\title{
Raman Mapping Applied to Reveal Internal Structures of Natural Minerals
}

\author{
L. Nasdala* \\ * Institut für Geowissenschaften, Johannes Gutenberg-Universität, D-55099 Mainz, Germany
}

Natural minerals are typically characterized by heterogeneous internal structures such as oscillatory growth zoning, sector zoning or patchy recrystallization patterns [1,2]. The occurrence of such features may provide valuable information about the formation and alteration history of minerals and their host rocks. In this paper, the application of Raman mapping to the study of internal structures of natural minerals is discussed.

The ability of the confocal Raman technique to analyze micron-sized areas [3] has opened up new opportunities for the non-destructive investigation of geological samples. For Raman maps, a large number of full Raman spectra are obtained in succession, mostly using a software-controlled sample stage. Each spectrum is thus assigned to $\mathrm{x}$ - and $\mathrm{y}$-coordinates and corresponds to one pixel in the final image(s). The disadvantage of this technique is that $10^{3}-10^{4}$ pixels are necessary to get well resolved images. Obtaining such a high number of spectra results in an enormous demand for analytical time (typically several hours). Therefore, Raman mapping may perhaps not become a routinely used technique in geoscientific research, rather this technique will prove extremely valuable for the detailed investigation of samples of special interest. The above disadvantage of Raman mapping is more than compensated for by the facts that, in contrast to the direct Raman imaging technique, measurements can be done in the confocal mode and sound mathematical treatment of the spectral information is possible. Background correction (for instance if the Raman signal should be obscured by strong luminescence) can be done, and not only intensities by any Raman information (e.g., band intensity ratios, band integrals, band widths, background slopes) can be used for image generation. Also, multiple images can be generated from one data set by choosing different parameters. Raman mapping may thus contribute to the detailed in-situ characterization of the structural state of natural minerals.

A typical example is to reveal internal structures of natural zircon $\left(\mathrm{ZrSiO}_{4}\right)$ crystals. As this mineral incorporates non-formula elements above the wt-\% level only rarely in geologic environments, Raman spectral changes are predominantly due to the so-called metamictization (process of accumulation of structural damage caused by radioactive self-irradiation [4]). Raman mapping thus yields precise information on the distribution of the radiation damage within single crystals (Fig. 1). The second example discussed in detail in the talk are patterns of enhanced pressure and strain around inclusions of high pressure minerals inside natural diamond crystals (Fig. 2). Here, Raman mapping yields in-situ information that cannot be obtained with any other technique at present.

\section{References:}

[1] P.W.O. Hoskin, Geochim. Cosmochim. Acta 64 (2000) 1905.

[2] G. Vavra et al., Contrib. Mineral. Petrol. 134 (1999) 380.

[3] L. Markwort et al., Appl. Spectrosc. 49 (1995) 1411.

[4] L. Nasdala et al., Contrib. Mineral. Petrol. 141 (2001) 125.

[5] M.H. Grimsditch et al., Phys. Rev. B 18 (1978) 901. 


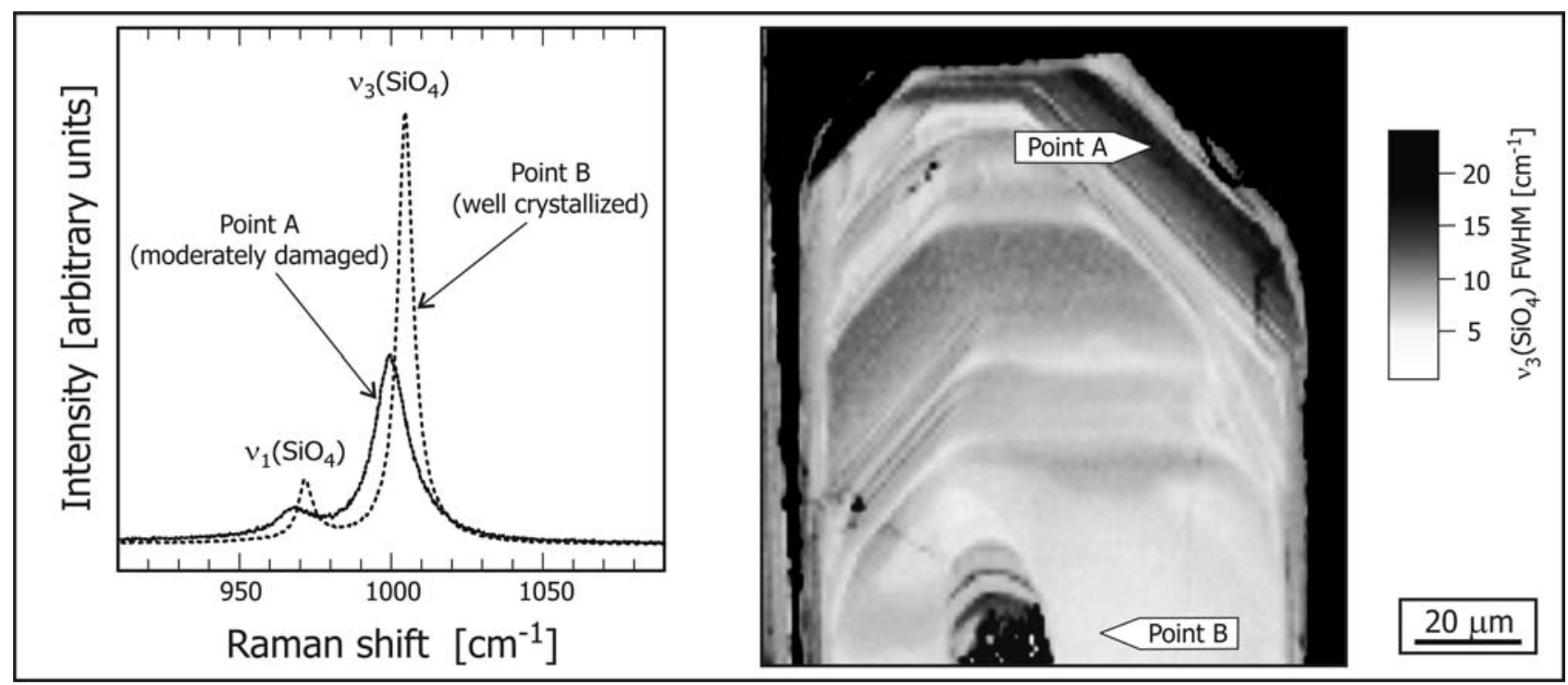

FIG. 1. Heterogeneous zircon crystal (Gold Butte, Nevada), showing primary growth zoning. Depending on their actinide ( $\mathrm{U}$ and $\mathrm{Th}$ ) content, micro-areas have experienced different selfirradiation doses. They are therefore characterized by different degrees of disturbance of their shortrange order. With increasing damage, Raman bands broaden and shift (shown left; cf. [4]). The full width at half band maximum (FWHM) of the $v_{3}\left(\mathrm{SiO}_{4}\right)$ mode is used to estimate the radiation damage. Plotting this parameter (shown right) yields a highly resolved "map of the crystallinity".

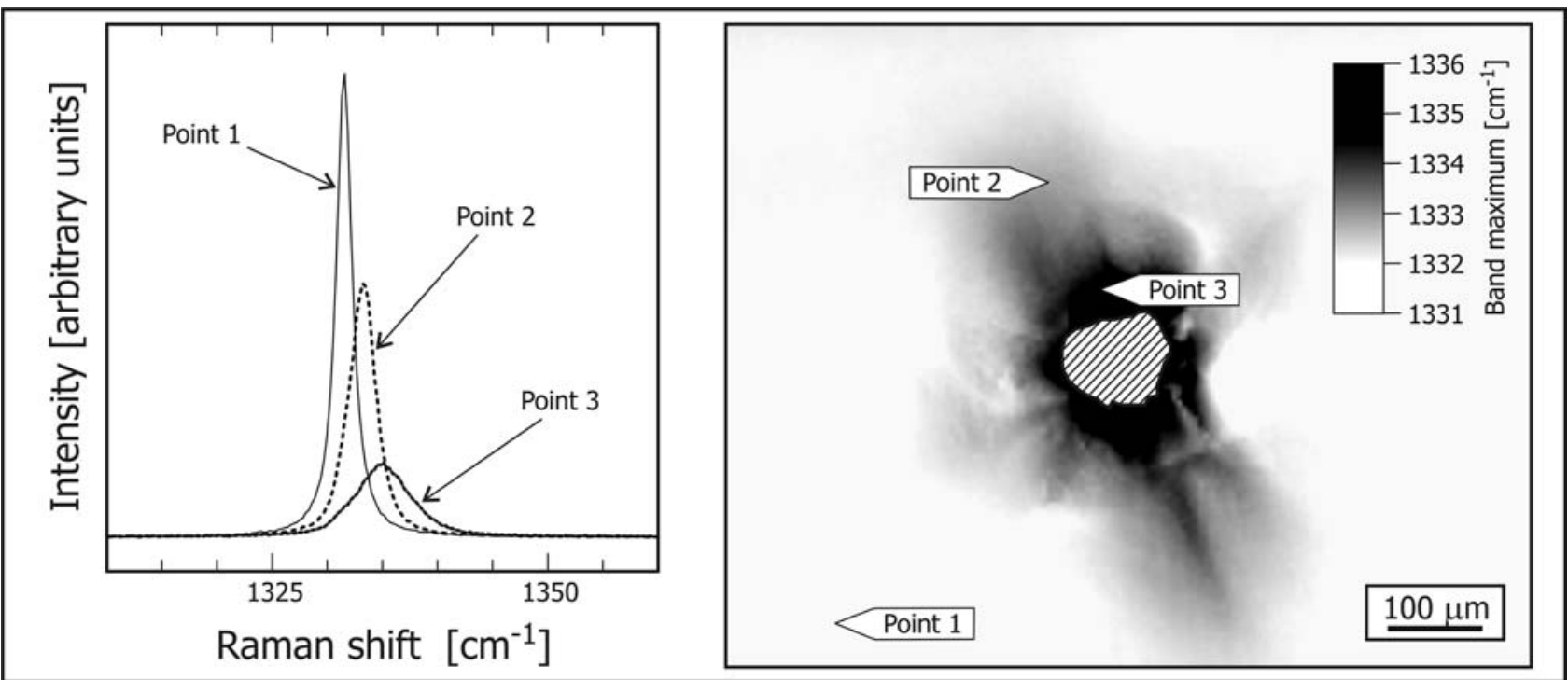

FIG.2. Inclusion of high pressure silicate minerals in a large diamond crystal (Guinea). The diamond has formed at high pressure in the earth mantle. Close to the inclusion (located $\sim 150 \mu \mathrm{m}$ behind the polished surface), the diamond structure is affected by residual pressure and additional strain, causing band shift and broadening (left part, cf. [5]). The map generated through plotting the Raman shift of the diamond $\mathrm{LO}=\mathrm{TO}$ mode (shown right) visualizes the lateral extension of the halo of enhanced pressure (the extension of the inclusion is shown hatched). 